\title{
Bandwidth Enhancement of Wireless Optical Communication Link Using a Near-Infrared Laser over Turbid Underwater Channel
}

\author{
It Ee Lee, ${ }^{1,2}$ Yujian Guo, ${ }^{1}$ Tien Khee Ng, ${ }^{1}$ Ki-Hong Park, ${ }^{1}$ Mohamed-Slim Alouini, ${ }^{1}$ and Boon S. Ooi ${ }^{1,}$ \\ ${ }^{1}$ Computer, Electrical and Mathematical Sciences and Engineering (CEMSE) Division \\ King Abdullah University of Science and Technology (KAUST) \\ Thuwal, Kingdom of Saudi Arabia \\ ${ }^{2}$ Faculty of Engineering, Multimedia University, Cyberjaya, Malaysia \\ "boon.ooi@kaust.edu.sa
}

\begin{abstract}
Underwater wireless optical communication (UWOC) has been widely studied as a promising alternative to establish reliable short-range marine communication links. Microscopic particulates suspended in various ocean, harbor and natural waters will alter the propagation characteristics of the optical signals underwater. In this paper, we demonstrate a gigabit nearinfrared (NIR)-based UWOC link using an 808-nm laser diode, to examine the feasibility of the proposed system in mitigating the particle scattering effect over turbid waters. We show that the NIR wavelengths presents greater resilience to the aqueous suspension of these micro-sized particles with a smaller scattering effect due to its longer wavelength, as evident by the smaller variations in the optical beam transmittance. It is also observed that the error performance is improved at higher concentrations albeit the significant reduction in received signal power. We further demonstrate that the overall frequency response of the system exhibits a bandwidth enhancement up to a few tens of $\mathrm{MHz}$ with increasing concentrations.
\end{abstract}

Keywords-water absorption; particle scattering; turbid media; frequency response; underwater wireless optical communication

\section{INTRODUCTION}

In the recent years, there has been an increasing demand for high-speed and long-distance underwater wireless data transmission, in order to support numerous bandwidthintensive marine research and infrastructures for the purposes of environmental surveillance, offshore exploration, disaster precaution, and military operations [1], [2]. Considering the bandwidth limitations of the acoustic [3] and radio frequency (RF) [4] communication technologies, underwater wireless optical communication (UWOC) presents a highly attractive and viable alternative, which utilizes optical laser beam waves as the transmission carriers for realizing a reliable, robust and high-speed wireless data transmission over the underwater channel [2]. This is mainly due to the abundant technical advantages of the UWOC technology, which include larger bandwidth with very high aggregate capacity, higher data rate, lower time latency, higher security, lower power consumption, and simplicity of system design with lower computational complexities [1], [2], [5].
The propagation of optical signal over the underwater channel suffers from severe water absorption and particle scattering, which results in the overall attenuation and multipath propagation of the optical beam, respectively [6]. Absorption is an energy transfer process that is dependent upon the refractive index of the water medium, whereby photons lose their energy by converting into other forms, such as heat and photosynthesis. This would in turn result in the irreversible loss of optical intensity, causing the total propagation energy of light to decrease continuously, thereby limiting the link distance of the UWOC channel. For various water types with different concentrations of phytoplankton, ranging from very clear (oligotrophic) to highly turbid (eutrophic) waters, the total spectral absorption can range from almost identical to that of pure water to one which shows orders-of-magnitude greater absorption than pure water [6]. Scattering refers to the multiple deflection of light from its original path after traversing a large number of particles present in the transmission medium, which are composed of discrete electric charges such as electrons and protons. Such phenomenon is mainly caused by the particles of sizes comparable to the wavelength (diffraction), or by particulate matters with refractive indices different from that of the water (refraction). As a result of the particle-light interaction, these charges are set in oscillation where the net effect is manifested in the emission of secondary radiation to give the scattering effect.

In the presence of microscopic organic and/or inorganic particulates suspended in different ocean and turbid coastal or littoral harbor waters, multiple scattering causes severe temporal pulse spreading with increasing attenuation lengths, which in turn limits the $3-\mathrm{dB}$ channel bandwidth and strongly degrades the error performance of the UWOC system due to intersymbol interference (ISI) [2]. While prior experimental works [7]-[10] have demonstrated the feasibility of the UWOC systems using visible-light laser diodes (LDs) with emission wavelengths in the blue-to-green spectral regime under clear water condition, the effects of particulates in-suspension in turbid underwater environments on the system performance were never thoroughly examined. Recently in [5], $\mathrm{Xu}$ et al. numerically studied the feasibility of employing directly modulated red light LD in the UWOC system, where it was

Funding acknowledgment: KACST TIC R2-FP-008, and KAUST baseline funding BAS/1/1614- 01-01. 


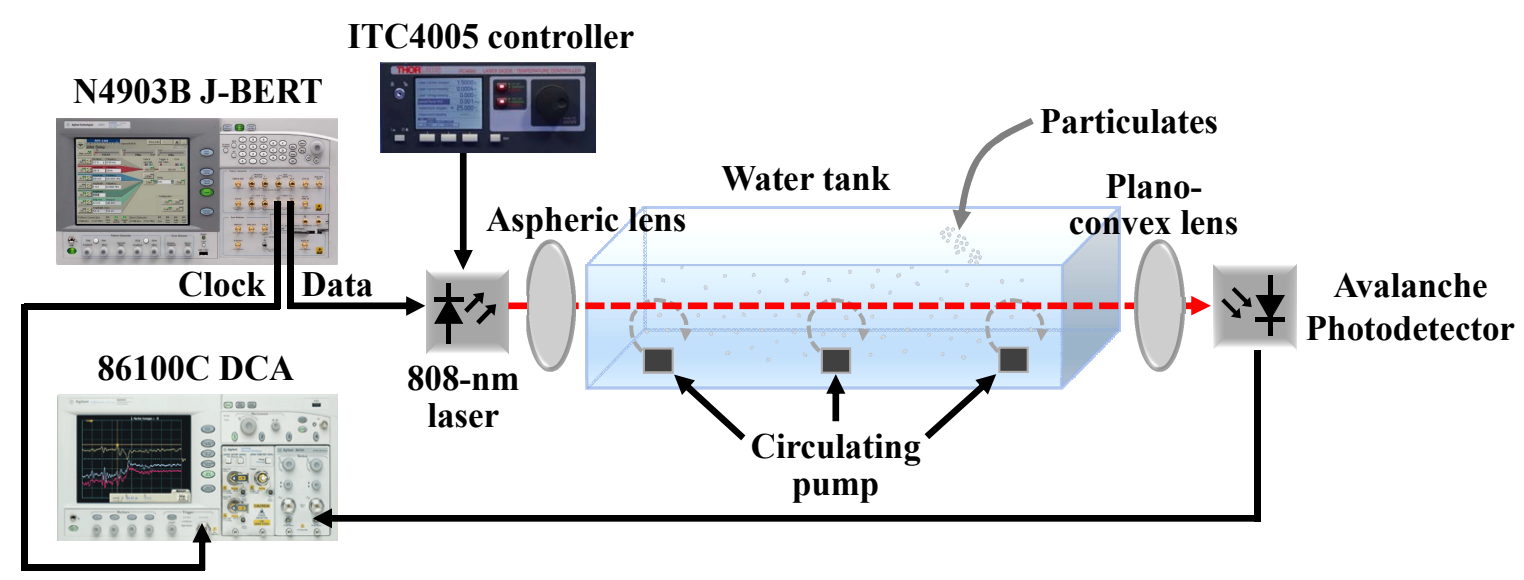

Figure 1. Schematic of the experimental setup for the NIR-based UWOC link using an 808-nm LD. Maalox solution is diluted in the water to emulate different turbidities of the underwater channel.

shown that the extinction coefficient of the red laser wavelength is smaller than the blue-green spectra within turbid waters. In addition, the channel bandwidth of red light is potentially enhanced due to the smaller scattering effect, thus revealing the advantages of using the longer-wavelength optical laser sources in mitigating the inherent multiple scattering and the possibility of exploring the near-infrared (NIR) regime for underwater communication.

In this paper, we examine the characteristics of the optical beams with wavelengths spanning the visible-light to NIR regime (300 $\mathrm{nm}$ to $900 \mathrm{~nm}$ ), under the influence of particulates in-suspension with increasing concentration in the water medium. Our results show that the blue-to-green $(400 \mathrm{~nm}$ to $550 \mathrm{~nm}$ ) visible wavelength region is highly susceptible to the presence of suspended particles, as evident from the relatively lower optical beam transmission (i.e., higher beam attenuation) due to significant scattering. The NIR regime is more resilient to the impacts of particle concentrations with a smaller scattering effect in the underwater channel due to its longer wavelength, as observed from the smaller variations in attenuation with increasing concentrations. Based upon a gigabit NIR-based UWOC experiment setup using an 808-nm $\mathrm{LD}$, we then investigate the resulting impact of particle concentrations on the performance of the NIR-based UWOC link in terms of the 3-dB channel bandwidth. It is demonstrated that the overall frequency response of the system is not deteriorated by the presence of particulates suspended within the underwater channel, but in turn exhibits a bandwidth enhancement up to a few tens of $\mathrm{MHz}$ with increasing turbidities. Therefore, the introduction of NIR LDs for underwater communication potentially enhances the system performance, particularly in highly turbid waters.

The remainder of this paper is organized as follows: Section 2 provides a detailed description of the NIR-based UWOC experiment setup for our proof-of-concept study and explains the methodology adopted for the proposed study here. In Section 3, we present our experimental results and discussions on the key observations and findings derived from this work. Finally, the concluding remarks are summarized in Section 4.

\section{THE EXPERIMENT SETUP}

Figure 1 depicts the schematic of the experimental setup for a single-input single-output (SISO) UWOC link employing the intensity modulation with direct detection (IM/DD) and the non-return-to-zero on-off-keying (NRZ-OOK) modulation. At the optical transmitter, a high-power $\varnothing 9 \mathrm{~mm}$ TO-can packaged NIR laser diode (LD) with a peak emission wavelength of 0.8 $\mu \mathrm{m}$ at a bias current of $383 \mathrm{~mA}$ is mounted on a thermoelectric cooler (TEC) module (Thorlabs LDM9T/M), and is driven by the Thorlabs ITC4005 LD and temperature controller. An aspheric lens (Thorlabs A110TM-B) with effective focal length of $6.24 \mathrm{~mm}$ and numerical aperture of 0.4 is attached to the TEC mount for collimating the optical beam. The pattern generator in the high-performance bit error rate (BER) tester (Agilent J-BERT N4903B) is used to generate the pseudorandom binary sequence (PRBS) $2^{10}-1$ pattern at different bit rates $R_{b}$, which is then modulated onto the instantaneous intensity of the optical beam wave.

The information-bearing collimated beam propagates through a 1.5-meter-long rectangular water tank $(0.3 \mathrm{~m}$ in width and $0.1 \mathrm{~m}$ in height) filled with fresh tap water to emulate the underwater channel. At the receiver side, a planoconvex lens (Thorlabs LA1027-B) with $25.4 \mathrm{~mm}$ diameter and $25.4 \mathrm{~mm}$ focal length is used to focus the optical signals onto the avalanche photodetector (APD) (Menlo Systems APD210). The overall frequency response of the system is measured using a parametric network analyzer (Agilent E8361C PNA). The performance of the NIR-based UWOC link is analyzed using the J-BERT and the digital communication analyzer (Agilent DCA-86100C), which measures the BER and eye diagram, respectively.

The turbidity of the underwater conditions are produced with suspensions of Aluminium hydroxide $\left(\mathrm{Al}(\mathrm{OH})_{3}\right)$ and Magnesium hydroxide $\left(\mathrm{Mg}(\mathrm{OH})_{2}\right)$, which are composites commonly found in a commercial antacid preparation $\left(\right.$ Maalox $\left.^{\circledR}\right)$. In different sets of experiment, the Maalox solution is added to the water tank containing 25.2 liters of water to obtain varying particle concentrations $N$. Circulating pumps are evenly placed along the channel to maintain homogeneity of the diluted Maalox solution in the water tank. In our effort to 
examine the characteristics of optical wavelengths at increasing water turbidities, different water samples with particle concentrations ranging from $0 \mathrm{~g} / \mathrm{m}^{3}$ (clear water) to $33.6 \mathrm{~g} / \mathrm{m}^{3}$ are prepared and held in quartz cuvettes. At room temperature, the optical transmittance spectra of these samples with varying Maalox concentrations are measured using a high performance spectrophotometer (Agilent Cary5000) spanning the ultraviolet (UV) to NIR wavelengths. To assess the optical characteristics of the $\mathrm{Al}(\mathrm{OH})_{3}$ and $\mathrm{Mg}(\mathrm{OH})_{2}$ aqueous suspension, the transmission spectra are obtained with respect to the base fluid and air reference, respectively.

\section{RESULTS AND DISCUSSIONS}

Figure 2 shows the overall optical transmittance spectra of the $\mathrm{Al}(\mathrm{OH})_{3}$ and $\mathrm{Mg}(\mathrm{OH})_{2}$ aqueous suspension with respect to the air, corrected for the reflectance term, at increasing particle concentrations $N$ from $0 \mathrm{~g} / \mathrm{m}^{3}$ (clear water) to $33.6 \mathrm{~g} / \mathrm{m}^{3}$. These plots depict the spectral transmittance of the system, as contributed by these compound microparticles and water (i.e., base fluid). Taking the clear water sample as a reference, it is evident that a near-ideal optical transmittance in excess of $99 \%$ is achieved for the violet-to-yellow ( $350 \mathrm{~nm}$ to $650 \mathrm{~nm}$ ) visible wavelength regime with a relatively flat transmission window, whereas the NIR $(>700 \mathrm{~nm})$ regime is subjected to significant attenuation resulting in a lower transmittance below $98 \%$. As the particle concentration increases, the optical transmittance exhibits an inherent downward shifting trend across the entire spectrum, in which the reduction in transmittance is particularly significant in the UV (40\% at $300 \mathrm{~nm})$ and visible regime (18\% at $450 \mathrm{~nm})$, as compared to the NIR regime with a smaller variation of $\sim 11 \%$ at the $808-\mathrm{nm}$ wavelength of interest. In the case of $N=23.52 \mathrm{~g} / \mathrm{m}^{3}$ and higher, it is observed that the transmittance of the blue-green visible wavelengths are comparable or lower than that of the 808-nm NIR wavelength, thus revealing the feasibility of the NIR-based UWOC link in turbid underwater environment.

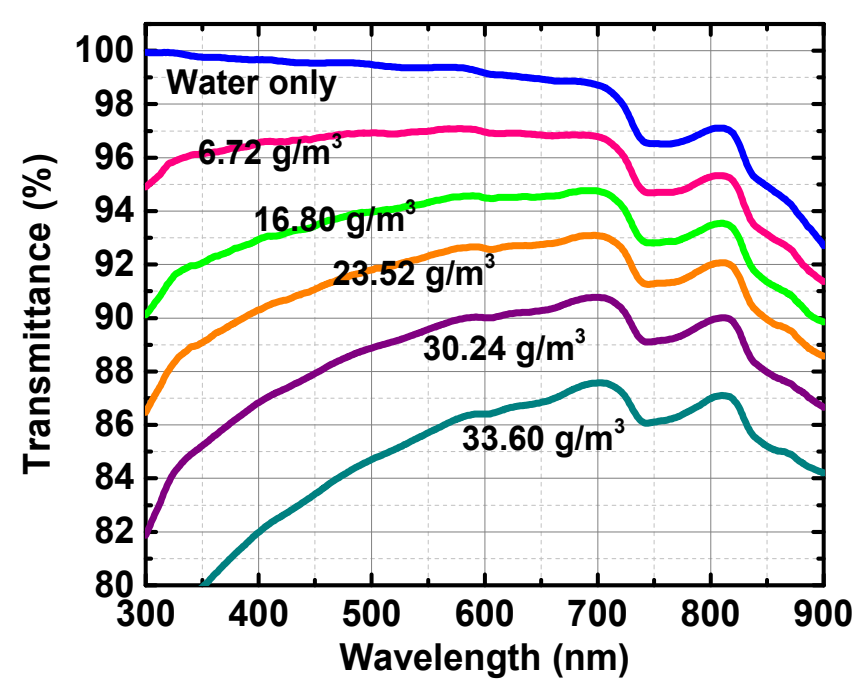

Figure 2. Overall optical transmittance spectra of the $\mathrm{Al}(\mathrm{OH})_{3}$ and $\mathrm{Mg}(\mathrm{OH})_{2}$ aqueous suspension, with concentrations ranging from $0 \mathrm{~g} / \mathrm{m}^{3}$ (clear water) to $33.6 \mathrm{~g} / \mathrm{m}^{3}$.

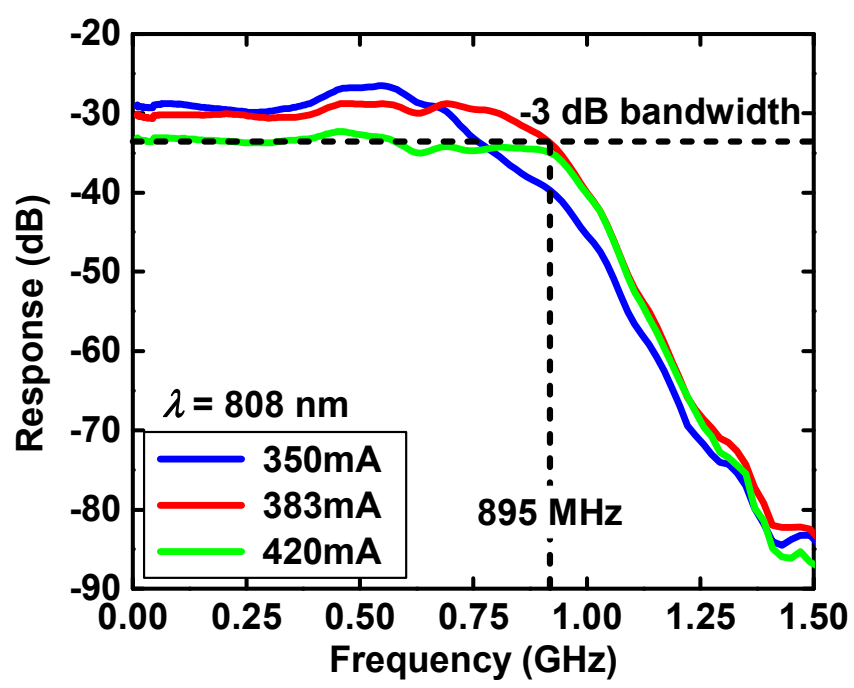

Figure 3. Overall frequency response of the NIR-based UWOC system at different biasing currents, which depicts the maximum $3 \mathrm{~dB}$ bandwidth occuring at $895 \mathrm{MHz}$.

As a figure of merit, the overall frequency response of the system is characterized under different biasing currents and depicted in Fig. 3, taking into account the LD and its driver, the underwater channel, and the APD. It is evident that the frequency response and modulation bandwidth are dependent upon the bias current, in which the bandwidth of the LD is extended with increasing current level from $350 \mathrm{~mA}$ to 420 $\mathrm{mA}$, due to the up-shift of the relaxation oscillation frequency and gain. The maximum $3 \mathrm{~dB}$ bandwidth attenuation occurs at approximately $895 \mathrm{MHz}$, as limited by the LD driver. Prior to the UWOC measurements and performance analysis, a throughput optimization of the system was conducted by adjusting the input to the LD, which includes the bias current level and modulation signal amplitude. The best achievable

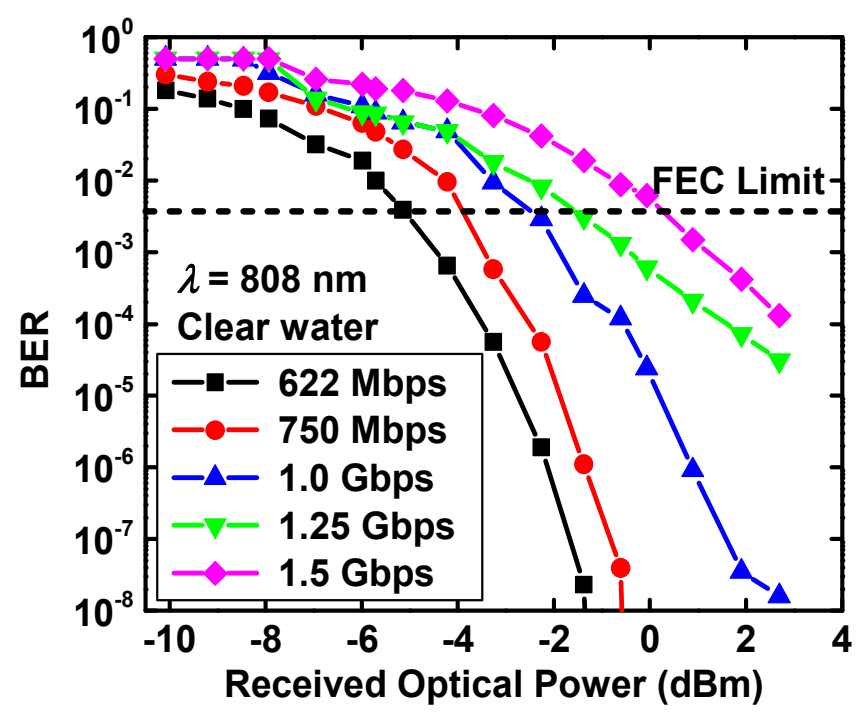

Figure 4. Measured BER as a function of the received optical power under a clear water condition at bit rates $R_{b}=\{0.62,0.75,1.0,1.25,1.5\}$ Gbps. 

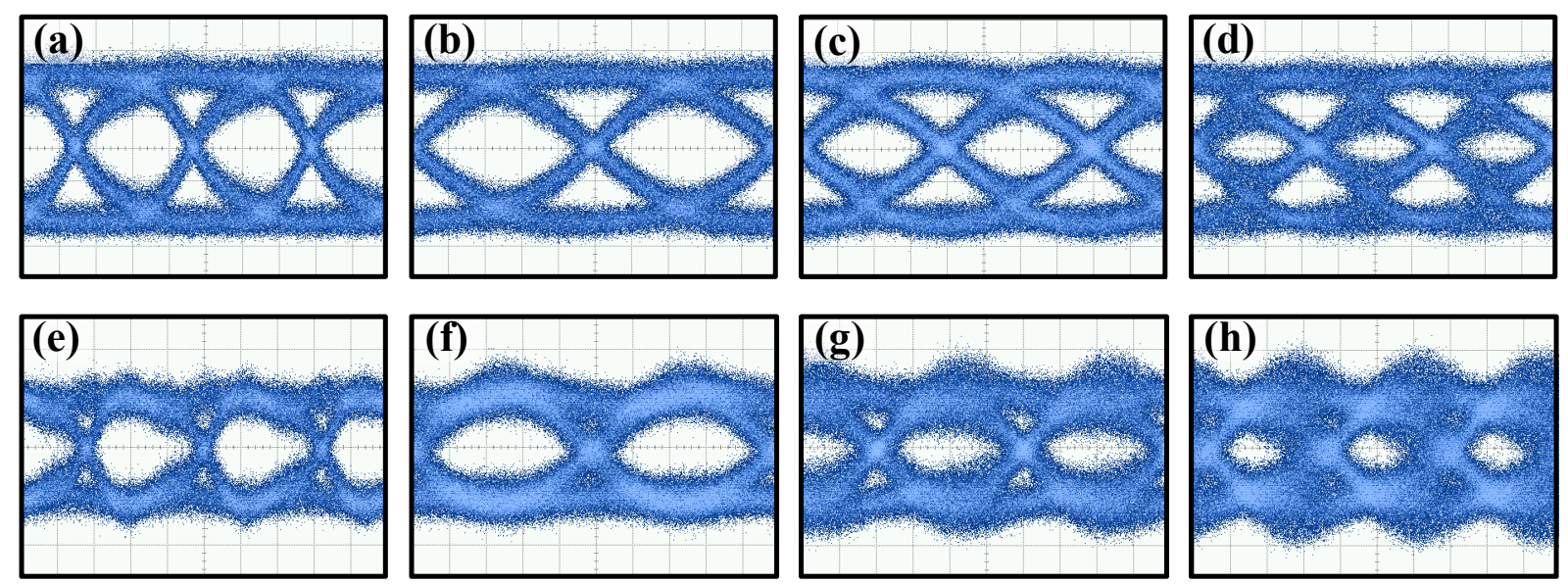

Figure 5. Measured eye diagrams at bit rates $R_{b}=\{0.62,1.0,1.25,1.5\}$ Gbps: (a)-(d) under clear underwater condition, and (e)-(h) for a more turbid underwater scenario with $N=11.33 \mathrm{~g} / \mathrm{m}^{3}$.

minimum BER is identified at an optimal bias current of 383 $\mathrm{mA}$ and modulation depth (i.e., peak-to-peak voltage) of $2.1 \mathrm{~V}$.

Figure 4 illustrates the measured BER performance as a function of the received optical power under a clear underwater condition at $R_{b}=\{0.62,0.75,1.0,1.25,1.5\} \mathrm{Gbps}$, with an estimated attenuation coefficient of $2.43 \mathrm{~m}^{-1}$. The received optical power was attenuated using a series of neutral density filter (Thorlabs NEK01) at the receiving-end. At a higher bit rate of $R_{b}=1.5 \mathrm{Gbps}$, the UWOC link is subjected to a higher power requirement of $0 \mathrm{dBm}$ to achieve the FEC limit criterion of $3.8 \times 10^{-3}$, as compared to $R_{b}=622 \mathrm{Mbps}$ with a relatively lower received optical power of $-5 \mathrm{dBm}$. The power penalty is attributed to the bandwidth limitation of the system. These observations are substantiated with the measured eye diagrams shown in Fig. 5(a)-(d), which depicts the narrowing of the

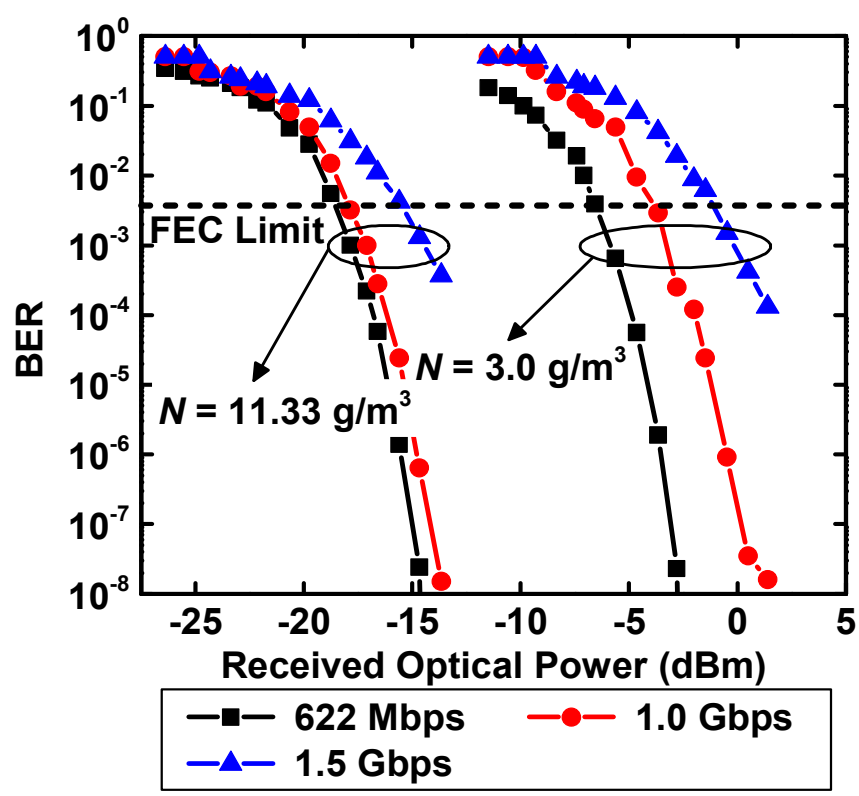

Figure 6. Measured BER in terms of the received optical power at bit rates $R_{b}=\{0.62,1.0,1.5\} \mathrm{Gbps}$, for different water turbidities with particle concentrations $N=\{3.0,11.33\} \mathrm{g} / \mathrm{m}^{3}$. open eye with increasing bit rates, thereby resulting in higher BER attainments. In Fig. 5(e)-(h), we examine the measured eye diagrams under a more turbid underwater scenario with $N$ $=11.33 \mathrm{~g} / \mathrm{m}^{3}$. It is evident that a comparable opening of the eye is maintained for the respective bit rates albeit the inherent signal attenuation from $2.77 \mathrm{dBm}$ to $-13.66 \mathrm{dBm}$, with an overall reduction of more than 16 order-of-magnitude.

In Fig. 6, we evaluate the effect of particle concentration on the error performance of the NIR-based UWOC link at different bit rates of $R_{b}=\{0.62,1.0,1.5\}$ Gbps. For a turbid underwater condition with $N=11.33 \mathrm{~g} / \mathrm{m}^{3}$, it is noticed that the UWOC link is capable of achieving the FEC limit criterion with a much smaller received optical power of $-18 \mathrm{dBm}$ at $R_{b}=$ $1.0 \mathrm{Gbps}$, as compared to the case of $N=3.0 \mathrm{~g} / \mathrm{m}^{3}$ with a significantly larger power requirement of $-4 \mathrm{dBm}$. Such observed trend is also depicted for the other considered $\mathrm{Rb}$ values, which can be explained by the fact that the NIR light with longer wavelength suffers from smaller scattering effect in turbid waters, and the forward scattering potentially directs the resultant beam towards the receiver.

The resultant impact of particle concentrations on the performance of the NIR-based UWOC system is also examined in terms of the 3-dB channel bandwidth, based upon the overall frequency response with increasing water turbidities, as shown in Fig. 7. In general, the bandwidth is typically maintained at $895 \mathrm{MHz}$ for the lower turbidity cases, whereas the frequency response exhibits a downward shifting trend with increasing concentrations due to the overall signal attenuation. In particular, it is observed that the $-3 \mathrm{~dB}$ bandwidth potentially increases to $941 \mathrm{MHz}$ at a higher concentration of $11.33 \mathrm{~g} / \mathrm{m}^{3}$. It is demonstrated that the overall frequency response of the system is not deteriorated by the presence of particulates suspended within the water channel, but in turn exhibits a bandwidth enhancement up to a few tens of $\mathrm{MHz}$ with increasing concentrations. In conclusion, the introduction of NIR LDs for underwater communication potentially enhances the error performance and channel bandwidth of the UWOC system, particularly in highly turbid underwater channel. 


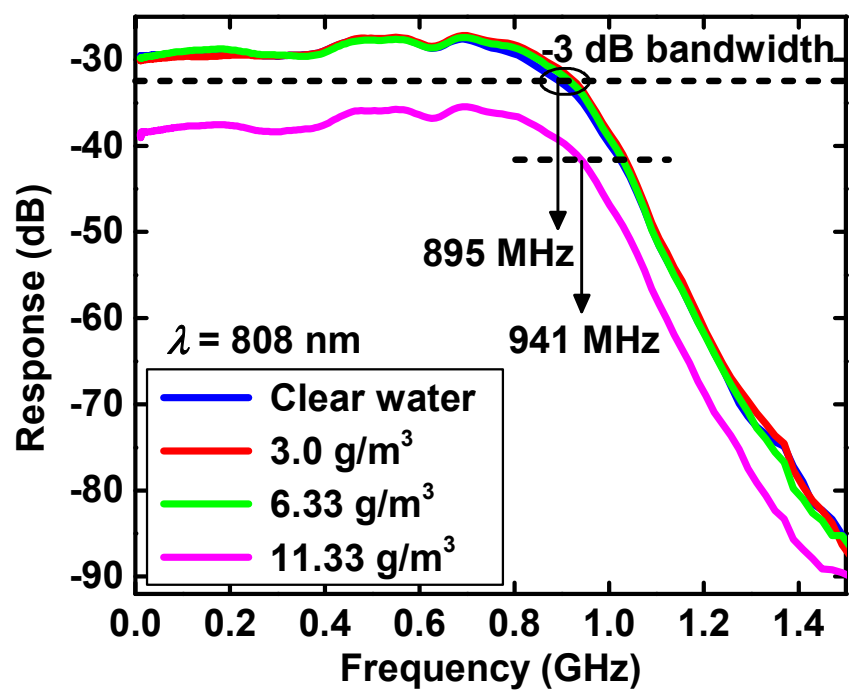

Figure 7. Variation of the overall frequency response with increasing water turbidities, depicting the enhancement of the $-3 \mathrm{~dB}$ bandwidth to $941 \mathrm{MHz}$ at a higher concentration of $11.33 \mathrm{~g} / \mathrm{m}^{3}$.

\section{CONCLUSIONS}

In summary, we have demonstrated the feasibility of a gigabit NIR-based underwater wireless transmission using an 808 -nm LD, and examined the system performance in the presence of particulate matters over turbid waters. It has been shown that the measured optical transmittance exhibits an inherent downward shifting trend across the entire spectrum at increasing particle concentrations, where significant reduction is evident in the UV and visible regime as compared to the NIR wavelengths. We have observed that the measured BER is improved with increasing concentrations albeit the inherent signal attenuation by more than 10-order-of-magnitude, as the water medium changes from a clear to high turbidity condition. The measured eye diagrams revealed that a comparable good opening of the eye is maintained for all the considered cases.
We have also demonstrated that the overall frequency response of the UWOC system is not deteriorated by the presence of particulates suspended within the underwater channel, but in turn exhibits a bandwidth enhancement up to a few tens of MHz. Therefore, UWOC links operating in the NIR regime with longer wavelengths are more resilient to the multiple scattering effects in highly turbid waters, and the forward scattering potentially directs the resultant beam towards the receiver, as evident by the enhancement of the bit error performance and system bandwidth.

\section{REFERENCES}

[1] H. Kaushal and G. Kaddoum, "Underwater optical wireless communication,” IEEE Access 4, 1518-1547 (2016).

[2] Z. Zeng, H. Zhang, Y. Dong, and J. Cheng, "A survey of underwater wireless optical communication,” IEEE Commun. Surveys Tuts. (2016).

[3] M. Stojanovic and J. Preisig, "Underwater acoustic communication channels: Propagation models and statistical characterization," IEEE Commun. Mag. 47, 84-89 (2009).

[4] X. Che, I. Wells, G. Dickers, P. Kear, and X. Gong, "Re-evaluation of RF electromagnetic communication in underwater sensor networks," IEEE Commun. Mag. 48, 143-151 (2010).

[5] J. Xu, Y. Song, X. Yu, A. Lin, M. Kong, J. Han, and N. Deng, "Underwater wireless transmission of high-speed QAM-OFDM signals using a compact red-light laser," Opt. Express 24, 8097-8109 (2016).

[6] C. D. Mobley, "The optical properties of water" in Handbook of optics, M. Bass, ed. (McGraw-Hill, 1995).

[7] H. M. Oubei, C. Li, K.-H. Park, T. K. Ng, M.-S. Alouini, and B. S. Ooi, "2.3 Gbit/s underwater wireless optical communications using directly modulated $520 \mathrm{~nm}$ laser diode," Opt. Express 23, 20743-20748 (2015).

[8] C. Shen, Y. Guo, H. M. Oubei, T. K. Ng, G. Liu, K.-H. Park, K.-T. Ho, M.-S. Alouini, and B. S. Ooi, "20-meter underwater wireless optical communication link with 1.5 Gbps data rate," Opt. Express 24, 2550225509 (2016).

[9] H. H. Lu, C. Y. Li, H. H. Lin, W. S. Tsai, C. A. Chu, B. R. Chen, and C. J. Wu, "An $8 \mathrm{~m} / 9.6 \mathrm{Gbps}$ underwater wireless optical communication system,” IEEE Photon. J. 8, 1-7 (2016).

[10] K. Nakamura, I. Mizukoshi, and M. Hanawa, "Optical wireless transmission of $405 \mathrm{~nm}, 1.45 \mathrm{Gbit} / \mathrm{s}$ optical IM/DD-OFDM signals through a $4.8 \mathrm{~m}$ underwater channel," Opt. Express 23, 1558-1566 (2015). 